\title{
ORIGINAL
}

\section{Safety and tolerability of non-neutralizing adrenomedullin antibody adrecizumab (HAM8101) in septic shock patients: the AdrenOSS-2 phase 2a biomarker-guided trial}

Pierre-François Laterre ${ }^{1}$, Peter Pickkers ${ }^{2}$, Gernot Marx ${ }^{3}$, Xavier Wittebole ${ }^{4}$, Ferhat Meziani ${ }^{5,6}$, Thierry Dugernier ${ }^{7}$, Vincent Huberlant ${ }^{8}$, Tobias Schuerholz ${ }^{9}$, Bruno François ${ }^{10}$, Jean-Baptiste Lascarrou ${ }^{11}$, Albertus Beishuizen ${ }^{12}$, Haikel Oueslati ${ }^{13}$, Damien Contou ${ }^{14}$, Oscar Hoiting ${ }^{15}$, Jean-Claude Lacherade ${ }^{16}$, Benjamin Chousterman ${ }^{17}$, Julien Pottecher ${ }^{18}$, Michael Bauer ${ }^{19,20}$, Thomas Godet ${ }^{21}$, Mahir Karakas ${ }^{22}$, Julie Helms ${ }^{23}$, Andreas Bergmann ${ }^{24}$, Jens Zimmermann ${ }^{24}$, Kathleen Richter ${ }^{24}$, Oliver Hartmann ${ }^{24}$, Melanie Pars ${ }^{24}$ and Alexandre Mebazaa ${ }^{25,26^{*}}$ (i) on behalf of the AdrenOSS-2 study participants

(c) 2021 Springer-Verlag GmbH Germany, part of Springer Nature

\begin{abstract}
Purpose: Investigate safety and tolerability of adrecizumab, a humanized monoclonal adrenomedullin antibody, in septic shock patients with high adrenomedullin.

Methods: Phase-2a, double-blind, randomized, placebo-controlled biomarker-guided trial with a single infusion of adrecizumab (2 or $4 \mathrm{mg} / \mathrm{kg}$ b.w.) compared to placebo. Patients with adrenomedullin above $70 \mathrm{pg} / \mathrm{mL}$, $<12 \mathrm{~h}$ of vasopressor start for septic shock were eligible. Randomization was 1:1:2. Primary safety (90-day mortality, treatment emergent adverse events (TEAE)) and tolerability (drug interruption, hemodynamics) endpoints were recorded. Efficacy endpoints included the Sepsis Support Index (SSI, reflecting ventilator- and shock-free days alive), change in Sequential-related Organ Failure Assessment (SOFA) and 28-day mortality.

Results: 301 patients were enrolled (median time of $8.5 \mathrm{~h}$ after vasopressor start). Adrecizumab was well tolerated (one interruption, no hemodynamic alteration) with no differences in frequency and severity in TEAEs between treatment arms (TEAE of grade 3 or higher: $70.5 \%$ in the adrecizumab group and $71.1 \%$ in the placebo group) nor in 90-day mortality. Difference in change in SSI between adrecizumab and placebo was $0.72(\mathrm{Cl}-1.93-0.49, p=0.24)$. Among various secondary end points, delta SOFA score (defined as maximum versus minimum SOFA) was more pronounced in the adrecizumab combined group compared to placebo [difference at $0.76(95 \% \mathrm{Cl} 0.18-1.35) ; p=0.007$ ]. 28-day mortality in the adrecizumab group was $23.9 \%$ and $27.7 \%$ in placebo with a hazard ratio of 0.84 (95\% confidence interval $0.53-1.31$, log-rank $p=0.44$ ).
\end{abstract}

\footnotetext{
${ }^{*}$ Correspondence: alexandre.mebazaa@aphp.fr

${ }^{26}$ Département d'Anesthésie-Réanimation, Hôpital Lariboisière, 2 Rue A Paré, 75010 Paris, France

Full author information is available at the end of the article

Pierre-François Laterre, Peter Pickkers and Gernot Marx contributed equally.
}

The AdrenOSS-2 study participants are listed in Acknowledgements. 
Conclusions: Overall, we successfully completed a randomized trial evaluating selecting patients for enrolment who had a disease-related biomarker. There were no overt signals of harm with using two doses of the adrenomedullin antibody adrecizumab; however, further randomized controlled trials are required to confirm efficacy and safety of this agent in septic shock patients.

Keywords: Septic shock, Adrenomedullin, Endothelial function, Adrecizumab (HAM8101), Enibarcimab

\section{Introduction}

Septic shock is a subset of sepsis in which underlying circulatory and cellular/metabolic abnormalities are profound enough to substantially increase mortality [1]. According to recent studies, vascular endothelial hyperpermeability and edema typically develop very early and involve the global endothelial barrier [2]. Adrenomedullin (ADM) is essential for endothelial barrier development and stability [3]. In human sepsis, various processes stimulate ADM secretion [4-6]. Bioactive ADM (bio-ADM) is an active, short-lived circulating peptide that derives from the prohormone fragment proADM. Upon admission to the intensive care unit (ICU), sepsis patients who are prone to develop septic shock, present elevated plasma levels of bio-ADM, the latter being associated with severe (multiple) organ dysfunction and an elevated mortality risk [7-9]. This bio-ADM increase in plasma concentration has been interpreted as both a bystander of endothelial dysfunction and a counterregulatory response to the impairment of vascular integrity [10]. Pre-clinical models of septic shock showed that modulating the ADM pathway with the high-affinity, non-neutralizing monoclonal anti-ADM antibody adrecizumab, improved endothelial homeostasis, organ function and survival [11-14]. Thus, strategies aiming at preventing disruption or at repairing endothelial cell-cell junctions may provide septic shock patients the opportunity to mitigate organ dysfunction and thereby to improve their outcome.

Conducting research in critically ill patient populations is challenging. Due to the heterogeneity of the populations, experts recently advised the use of advanced trial designs to assess and promote "personalized" therapies $[15,16]$. Such trial designs combine biomarker-guided enrichment of enrolled patients to reduce heterogeneity and biomarker-guided therapy to enhance likelihood of success for novel therapies. The latter are based on the biological plausibility for the new compound to exert therapeutic efficacy.

We now report the results of the AdrenOSS-2 trial, a phase $2 \mathrm{a}$, randomized, double-blind, placebo-controlled trial using bio-ADM-guided enrichment and therapy with the non-neutralizing anti-ADM antibody adrecizumab in early septic shock patients.

\section{Take-home message}

The primary endpoint of AdrenOSS-2 was achieved as adrecizumab was well tolerated and showed a favorable safety profile. In this biomarker-guided trial, the mode of action was confirmed: adrecizumab rapidly increased plasma levels of bioactive adrenomedullin, a key hormone restoring and maintaining vascular integrity and endothelial function.

\section{Methods}

\section{Design}

This was a double-blind, placebo-controlled, randomized, multicenter, biomarker-guided and dose-finding phase 2a trial to investigate the safety, tolerability, efficacy and pharmacokinetics of adrecizumab in patients with early septic shock and elevated bio-ADM levels. The trial was conducted in thirty hospitals with medical, surgical and/or mixed ICUs in Belgium, France, Germany and the Netherlands. Further details on the trial setting and design were reported earlier [17]. Of note, preliminary results were presented at the ISICEM meeting.

\section{Ethical considerations}

The trial procedures and the informed consent form (ICF) process were approved by the respective independent ethics committee (IEC) following international standards and national requirements of each participating country. The AdrenOSS-2 trial was registered at Clinical trial.gov (NCT03085758).

\section{Participants}

Patients ( $\geq 18$ years) at the early phase of septic shock who met the inclusion- and none of the exclusion criteria were enrolled. Main inclusion criteria were elevated bio-ADM values $(>70 \mathrm{pg} / \mathrm{mL})$, in the early phase of septic shock, defined as start of vasopressor therapy less than $12 \mathrm{~h}$ before inclusion. A full list of inclusion and exclusion criteria can be found in the electronic supplementary material. After a written informed consent form was provided by the patient or his/her legal representative and when plasma bio-ADM concentration was $>70 \mathrm{pg} /$ $\mathrm{mL}$ (Sphingotest ${ }^{\circledR}$ bio-ADM ${ }^{\circledR}$, SphingoTec GmbH, Hennigsdorf, Germany), a clinical coordination center (CCC) confirmed patient eligibility for enrolment in the trial. All trial related data was captured in a pseudonymized way. 
For bio-ADM measures, blood was withdrawn in EDTA tubes and immediately centrifuged at room temperature. Bio-ADM measures followed 3 phases that usually took less than $2 \mathrm{~h}$ : (1) reconstitute controls and tracer, (2) incubation for $1 \mathrm{~h}$ at $22{ }^{\circ} \mathrm{C}$ and (3) wash and measurement of the luminescence signals by the microplate Centro LB960. For each patient, Bio-ADM was performed, once at screening, locally either in the laboratory or directly on ICU by trained staff or, for Paris and surrounding region, by a central laboratory available 24/7, in Lariboisière hospital. Of note, no bio-ADM measure was allowed after the initiation of study drug. The threshold of $70 \mathrm{pg} / \mathrm{mL}$ on bio-ADM was selected based on increased risk of bad outcome described in previous studies [8].

\section{Intervention}

Patients were randomly assigned in a 1:1:2 ratio to either treatment arm A (adrecizumab $2 \mathrm{mg} / \mathrm{kg}$ body weight (b.w.)), treatment arm B (adrecizumab $4 \mathrm{mg} / \mathrm{kg}$ b.w.) or placebo, respectively. Patients received the assigned trial medication in a single intravenous infusion (duration: approximately $1 \mathrm{~h}$ ) [17].

A nested pharmacokinetic (PK) sub-study was performed in a subgroup of 56 patients for whom the complete dataset of all 7 samples were available, with blood sampling from Day 1 to Day 7 and on Day 28 to determine the pharmacokinetic profile of adrecizumab. Blood samples were taken at fixed time points prior to and following trial medication administration (see trial protocol at ClinicalTrials.gov Identifier: NCT03085758). The first blood sample was taken more than $20 \mathrm{~h}$ after initiation of studied therapy in three patients that were excluded from this analysis. Thus, final analysis was based on 53 patients.

\section{Outcome measures}

The primary objective of this study was to investigate safety and tolerability of adrecizumab. Endpoints of safety were frequency and severity of adverse events (AEs, recorded thoroughly starting from informed consent form signature) and treatment emergent adverse events (TEAEs) and 90-day mortality and endpoints of tolerability were blood pressure, heart rate, and episodes of interruption of infusion. Secondary objectives were clinical efficacy and pharmacokinetics of adrecizumab. Key efficacy endpoint was the Sepsis Support Index (SSI) defined as days with organ support (hemodynamic, renal or respiratory) or death within 14-day follow-up, achieving a combined endpoint of organ dysfunction and death. A detailed description on how to calculate the Index is described by Geven et al. [17] and in the electronic supplementary material. Other secondary efficacy endpoints included Day 28 mortality, change in SOFA score (assessed every $24 \mathrm{~h}$ during the first week), duration of ICU stay, changes of vital signs [e.g. mean arterial pressure (MAP) and heart rate (HR)], as well as other parameters collected during ICU stay such as fluid balance, partial pressure of oxygen/fraction of inspired oxygen $\left(\mathrm{PaO}_{2} / \mathrm{FiO}_{2}\right)$, creatinine, procalcitonin (PCT) level, duration of vasopressor use and Quality of Life (Euro-QoL-5). In the nested pharmacokinetic sub-study, the following parameters were determined: peak observed maximum plasma concentrations $\left(C_{\max }\right)$, time to $C_{\max }\left(t_{\max }\right)$, systemic exposure, area under the curve (AUC), volume of distribution $(V)$, systemic clearance (CL) and elimination half-life $\left(t^{1 / 2}\right)$.

\section{Data analyses and statistics}

Safety and tolerability endpoints were reported and efficacy endpoints were analyzed in all patients who were randomized to a treatment group (Intent-to-treat (ITT) analysis). In the ITT data set, all patients provided informed consent and received the trial medication. The primary analysis included the comparison of the combined adrecizumab doses to placebo [17]. The per-protocol (PP) analysis included all patients who, in addition, received the trial medication according to the protocol with minor deviations only and satisfied all major entry criteria. For the pharmacokinetic analysis set geometric means and standard deviation (SD) were calculated, for each time point and dose, if $2 / 3$ of the values were above the lower limit of quantification and if $\mathrm{n}$ was greater than two. Demographic and medical background data, safety variables and secondary endpoints are to be analyzed by means of descriptive and exploratory methods. Continuous data were analyzed by number of patients, median and interquartile range. Discrete data (i.e. metric, but non-continuous data, like SSI, SOFA score, and Quality of Life), were analyzed by number of patients, arithmetic mean and SD. Exploratory comparisons for both continuous and discrete data were performed using the Kruskal-Wallis test. Categorial variables are summarized category-wise giving numbers and percentages, and compared using the $\mathrm{Chi}^{2}$ test for contingency tables. Since antibodies were given in excess in both treatment arms (2 and $4 \mathrm{mg} / \mathrm{kg}$ ), data were combined for efficacy analysis. The distribution of the SSI in placebo and the combined adrecizumab arms was compared via the KolmogorovSmirnov test and the empirical distribution function was used for comparison. The delta SSI was estimated and tested using both the $\mathrm{t}$ test and the Kruskal-Wallis test with the Hodges-Lehmann estimator. The log-rank test was chosen for showing differences in mortality rates among treatment groups, and Kaplan-Meier plots were used for illustration. Unadjusted and adjusted hazard 
ratios (HR) were estimated via Cox proportional hazard models. HR were adjusted for possible differences in baseline severity (pre-Investigational Medicinal Product (pre-IMP) SOFA and APACHE score, and pre-IMP bio-ADM and lactate), as well as for baseline characteristics that differed between treatment arms. Adjusted HR were derived by including the respective co-variable into a multivariable Cox regression model, together with the treatment effect. Imputation for SOFA score were performed for patients discharged or dead on day 2-7 after enrolment (see electronic supplementary material) (Grooth et al. [18]). Note that these patients are not "missing at random", and therefore cannot be treated like other missing data points. No additional imputations were made for the primary analysis reported neither for other missing SOFA scores nor for any SSI. All reported $p$ values are 2 -sided. $p$ values below 0.05 were considered significant. This phase 2 trial was not powered to prove clinical efficacy. No confirmative testing was considered, and as secondary outcome parameters were in part correlated, especially for variables evaluated at multiple time points, reported $p$ values were not corrected for multiple testing. The sample size calculation was based on simulation of real patient data, described in the method paper [17] and in the electronic supplementary material. Statistical analyses were performed using SAS version 9.3, and R version 3.4.3 (http://www.r-project.org). The calculation of the PK parameters was performed using Phoenix ${ }^{\circledR}$ WinNonlin version 8.1.

\section{Results}

\section{Patients and study treatment}

Enrollment of patients started with the first patient being randomized on December 8, 2017. The last patient was randomized on September 25, 2019. Altogether, 459 patients were screened. Out of these 158 were non-eligible and, therefore, not randomized $(n=91$ with bio$\mathrm{ADM}<70 \mathrm{pg} / \mathrm{mL} ; n=67$ for failing with regard to other inclusion/exclusion criteria). In total, 301 patients were randomized to either placebo $(n=152), 2 \mathrm{mg} / \mathrm{kg}(n=72)$ or $4 \mathrm{mg} / \mathrm{kg}$ adrecizumab $(n=77)$, defining the ITT population (Fig. 1). For the PP analysis, seven patients were excluded, see Supplement Table 2 for details.

AdrenOSS-2 showed that all requirements, including local bio-ADM measures, were duable in early septic shock, allowing a study drug initiation 8.50 [5.83-11.00] hours after onset of shock (8.67 [5.58-10.95] hours in the adrecizumab group and 8.38 [6-10.87] hours in the placebo group). Baseline characteristics are shown in Table 1 and were broadly balanced among all groups in terms of age, mean body temperature, MAP and respiratory rate. Sources of infection were predominantly abdomen (21.6\%), lung (20.9\%) or urinary tract $(17.9 \%)$.

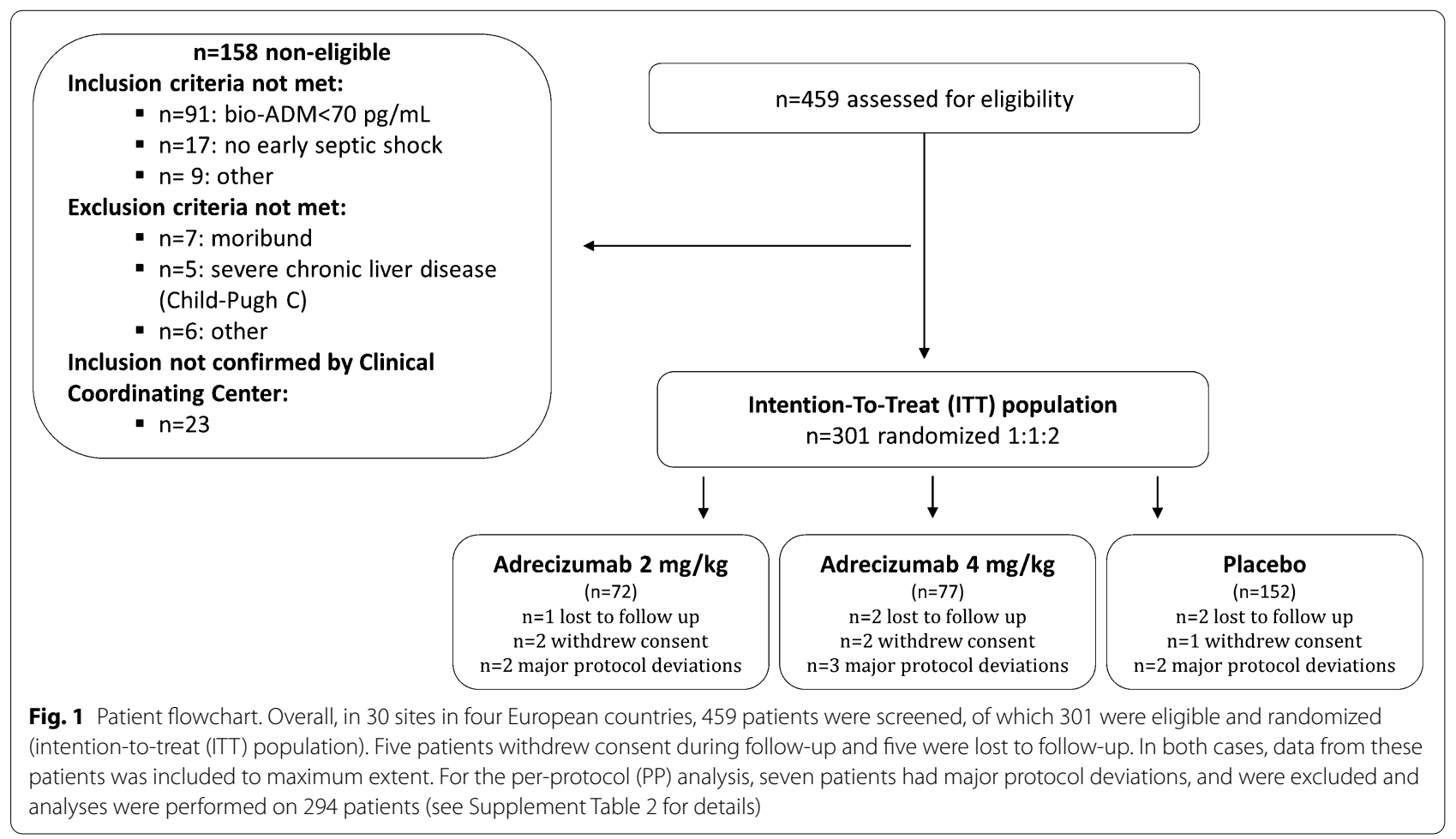


Table 1 Patient characteristics for all patients randomized (ITT, $n=301)$

\begin{tabular}{|c|c|c|c|c|c|}
\hline Variable & $n$ & $\begin{array}{l}\text { Adrecizumab } \\
2 \mathrm{mg} / \mathrm{kg}(n=72)\end{array}$ & $\begin{array}{l}\text { Adrecizumab } \\
4 \mathrm{mg} / \mathrm{kg}(n=77)\end{array}$ & $\begin{array}{l}\text { Placebo } \\
(n=152)\end{array}$ & $\begin{array}{l}\text { Adrecizumab } \\
(n=149)\end{array}$ \\
\hline Age (years) & 301 & $68[59-75]$ & $71[65-77]$ & $71[60-78]$ & $71[62-76]$ \\
\hline Gender female & 301 & $27(37.5)$ & $24(31.2)$ & $66(43.4)$ & $51(34.2)$ \\
\hline BMI $\left(\mathrm{kg} / \mathrm{m}^{2}\right)$ & $299^{a}$ & $25.2[22.9-28.1]$ & $25.6[23.4-29.5]$ & $26.5[24.1-30.4]$ & $25.3[23.2-29]$ \\
\hline \multicolumn{6}{|l|}{ Parameters at inclusion } \\
\hline Temperature $\left({ }^{\circ} \mathrm{C}\right)$ & $284^{a}$ & $36.9[36.3-37.6]$ & 37 [36.2-37.7] & 37.1 [36.5-37.6] & $37[36.25-37.7]$ \\
\hline HR (bpm) & 301 & $102[86-123]$ & $97[83-111]$ & $96[79-110]$ & $98[84-115]$ \\
\hline MAP $(\mathrm{mmHg})$ & 301 & $72[66-80.25]$ & $70[66-76]$ & $71[66-79]$ & $71[66-78]$ \\
\hline SOFA (points) & $254^{a}$ & $11[7-11]$ & $10[7-11]$ & $9[7.75-11.25]$ & $10[7-11]$ \\
\hline APACHE II (points) & 272 & $32[29-34]$ & $33[28-36]$ & $33[29-36]$ & $32[28.75-35]$ \\
\hline Origin of sepsis ${ }^{b}$ & 301 & & & & \\
\hline Lung & 63 & $14(19.4)$ & $17(22.1)$ & $32(21.1)$ & $31(20.8)$ \\
\hline Peritonitis & 65 & $12(16.7)$ & $17(22.1)$ & $36(23.7)$ & $29(19.5)$ \\
\hline Urinary tract & 54 & $18(25)$ & $10(13)$ & $26(17.1)$ & $28(18.8)$ \\
\hline Skin and soft tissue & 24 & $2(2.8)$ & $8(10.4)$ & $14(9.2)$ & $10(6.7)$ \\
\hline Others & 95 & $26(36.1)$ & $25(32.5)$ & $44(28.9)$ & $51(34.2)$ \\
\hline \multicolumn{6}{|l|}{ Co-morbidities ${ }^{c}$} \\
\hline Chronic heart failure & 301 & $9(12.5)$ & $8(10.4)$ & $15(9.9)$ & $17(11.4)$ \\
\hline Hypertension & 301 & $35(48.6)$ & $45(58.4)$ & $101(66.4)$ & $80(53.7)$ \\
\hline \multicolumn{6}{|l|}{ Biomarker } \\
\hline Bio-ADM, local (pg/mL) & 301 & $210.8[127.1-318.4]$ & $203.2[131.9-372.6]$ & $199.9[120-300.1]$ & $205.1[128.2-339.3]$ \\
\hline \multicolumn{6}{|l|}{ Treatment on admission ${ }^{c}$} \\
\hline $\begin{array}{l}\text { Time ICU admission to studied therapies } \\
\text { (days) }\end{array}$ & 301 & $0.36[0.23-0.56]$ & $0.43[0.26-0.58]$ & $0.31[0.22-0.51]$ & $0.42[0.25-0.57]$ \\
\hline $\begin{array}{l}\text { Time between initiation of vasopressor and } \\
\text { initiation of studied therapies (h) }\end{array}$ & 301 & $7.48[5.5-10.49]$ & $9.63[5.67-11.17]$ & $8.38[6-10.87]$ & $8.67[5.58-10.95]$ \\
\hline Days on vasopressor, 14 days (days) & 297 & $4.14(2.94)$ & $5.09(3.82)$ & $4.63(3.44)$ & $4.68(3.86)$ \\
\hline Days on vasopressor, 28 days (days) & 297 & $4.82(4.65)$ & $5.97(5.54)$ & $5.41(5.14)$ & $5.27(5.11)$ \\
\hline
\end{tabular}

Median and interquartile range, mean and SD or counts and percentages are given. $p$ values from Chi $^{2}$ or Kruskal-Wallis test, as appropriate

$I Q R$ interquartile ranges; $B M I$ Body Mass Index; $H R$ heart rate; $b p m$ beats per minute; $M A P$ mean arterial pressure; $m m H g$ millimetres of Mercury; bio- $A D M$ biologically active adrenomedullin; $\mathrm{pg} / \mathrm{mL}$ picogram per milliliter; $M R$-proADM circulating mid-regional proADM; $n m o l / L$ nano-mol per liter; $n g / m L$ nano-gramme per millilitre;

SOFA Sepsis-related Organ Failure Assessment

a Missing values

b Only the top three origins of sepsis are presented

c Only a selection is presented here

SOFA and APACHE II scores were typical for a septic shock population at baseline: 9 [7-10] and 33 [29-36] (median [IQR]) in the placebo group and 10 [7-11] and 32 [29-35] in the combined Adrecizumab 2 and $4 \mathrm{mg} / \mathrm{kg}$ arms, respectively. (Table 1 ).

\section{Safety and tolerability of combined adrecizumab dose-groups}

Results of the primary safety (AEs and TEAEs) analysis are summarized in Fig. 2. The incidence of AEs and TEAEs was similar among the three studied arms. A total of 284/301 (97.3\%) patients experienced at least one TEAE (details by primary organ system class are given in the electronic supplementary material. Overall, 105 out of 149 patients reported TEAEs of grade 3 or higher
(70.5\%) in the combined adrecizumab group ( 2 and $4 \mathrm{mg} /$ $\mathrm{kg}$ ), and 108/152 (71.1\%) patients in the placebo group. A total of 25 patients $(8.3 \%)$ reported a TEAE related to the drug administration overall: $12(8.3 \%)$ in the combined adrecizumab group and 13 (8.6\%) in the placebo group. For one patient randomized to the $4 \mathrm{mg} / \mathrm{kg}$ adrecizumab group, the infusion was stopped prematurely due to a cardiac repolarization abnormality, observed during electrocardiography. Patient survival at Day 90 between the combined adrecizumab group and placebo was similar.

Concerning tolerability, infusion was stopped prematurely in one patient of the adrecizumab $4 \mathrm{mg} / \mathrm{kg}$ group and similar changes in arterial pressure or heart rate were seen over time between patients randomized to adrecizumab combined or placebo groups (Supplement Fig. 2). 


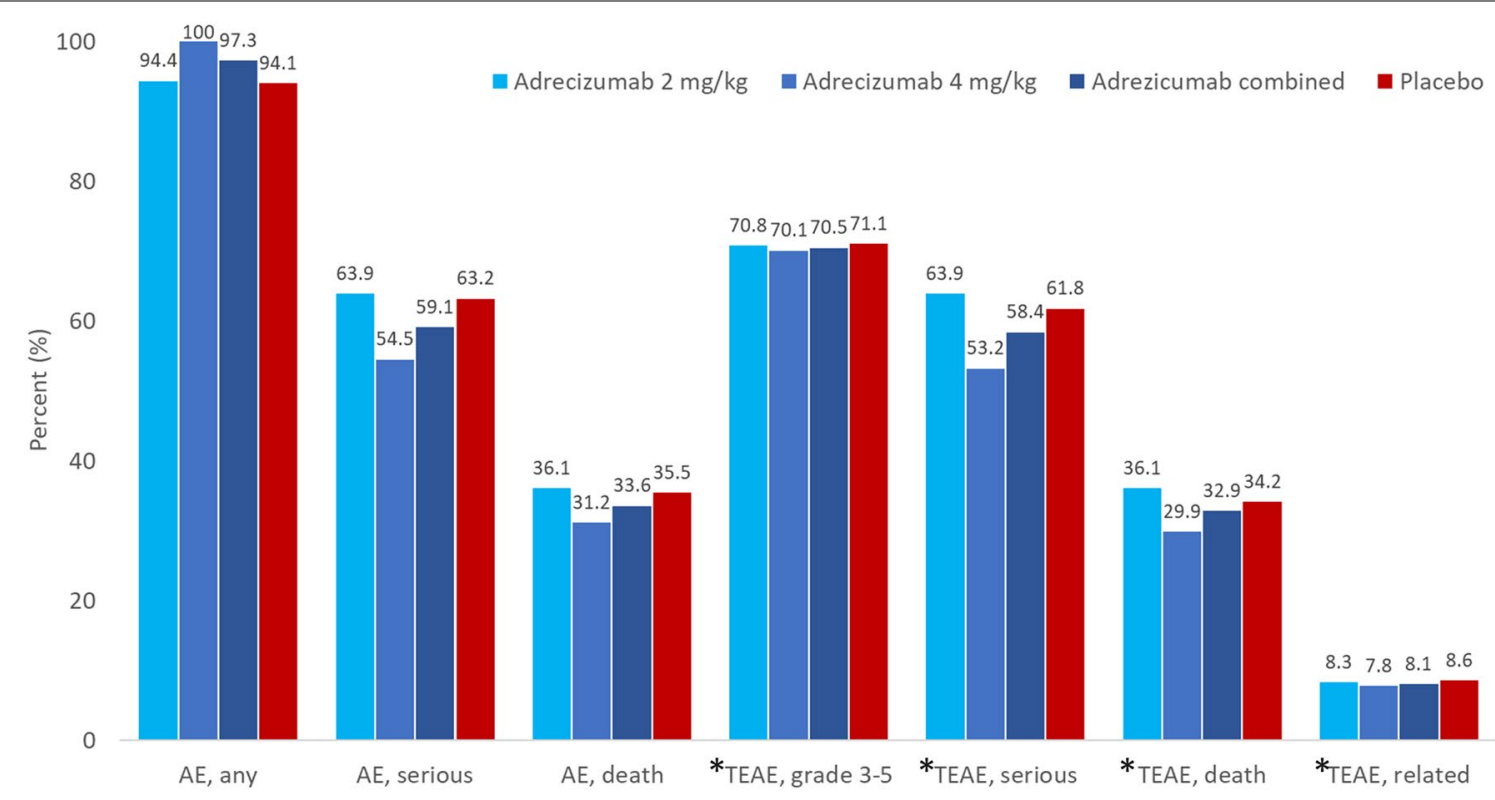

Fig. 2 Adverse events (AE) and Treatment Emergent Adverse Events (TEAEs) by treatment arm in all patients randomized (ITT, $n=301)$. TEAEs were AEs not present at baseline, or AEs that worsened after start of treatment even if they were present at baseline. AEs (any, serious or death) or TEAEs were similar, between patients randomized to Adrecizumab and placebo. ${ }^{*} T E A E$, grade $3-5$ all severe events according to Common Terminology Criteria for Adverse Events (CTCAE) classification; TEAE, serious all serious events; TEAE, death all events that led to death; TEAE, related all events that were categorized as related to Adrecizumab

\section{Efficacy of the combined adrecizumab group}

No significant treatment effect of the combined adrecizumab arms on the SSI within 14 days follow-up was observed, the difference in change in SSI between adrecizumab and placebo was 0.72 (95\% CI -1.93-0.49, $p=0.242$ ) based on the $t$ test and 0 (95\% CI $0-0, p=0.32$ ) based on Hodges-Lehmann. The SSI was below 14 in $60.3 \%$ of patients treated with placebo and in $58.9 \%$ in patients treated with adrecizumab (Fig. 3A). Results were similar for the SSI within the 28 days follow-up. Looking at each component separately, the respiratory, renal, cardiac, as well as the mortality component showed no differences between treatment arms. See Supplement Table 4A and B for full details.

Among various secondary endpoints, Fig. 3B shows that the delta SOFA score (defined as maximum versus minimum SOFA during the first $144 \mathrm{~h}$ after randomization, available in only $n=280$ due to missings) was more pronounced in the adrecizumab combined group compared to placebo (mean (SD) 4.05 (2.41) vs. 3.29 (2.56); difference in the delta was 0.76 (95\% CI $0.18-1.35) ; p=0.007$. The difference in the change in SOFA between adrecizumab combined and placebo (available only in up to $n=239$ patients due to missings) was 1.0 SOFA score points (mean, $95 \% \mathrm{CI}$ $0.0-2.0), 0.8$ (-0.5-2.2), 0.7 (-0.9-2.2), 1.0 (-0.6-2.7), 0.6 $(-1.1-2.5)$ and $0.5(-1.5-2.4)$ for the change from pre-IMP to $24,48,72,96,120$ and $144 \mathrm{~h}$, respectively (Supplement
Table 4A). Sensitivity analysis and histograms are shown in supplement Figs. 6 \& 7 .

The 28 -day mortality was $23.9 \%$ and $27.7 \%$ in the adrecizumab combined and placebo groups, respectively, with the unadjusted HR (95\% CI) of 0.84 [0.53-1.31], log-rank $p=0.44$ for adrecizumab combined (Fig. 4A, B). Results after adjustment for baseline severity (pre-IMP SOFA score or bio-ADM) are shown in Supplement Table 5. Exploratory interaction analysis for 28-day mortality did not indicate a further need for stratification based on initial bio-ADM beyond the initial inclusion criteria (interaction term $p$ value $=0.25$, model including treatment, baseline bio-ADM and the interaction term), nor on severity scores $(p=0.96$ and 0.75 for SOFA score or APACHE II score, respectively). On short-term mortality, 32 (21.3\%) patients died in the placebo group and $25(16.8 \%)$ in the adrecizumab combined group within the first 14 days, while from day $15-28$, in both placebo and adrecizumab combined group, another 10 patients died (6.6\% and 6.7\%, respectively, see Supplement Table $4 \mathrm{~B}$ for further details).

Concerning quality of life assessments, change of the Visual Analogue Scale (VAS) from ICU discharge to 90-day follow-up: $16.5 \pm 22.5$ compared to $7.6 \pm 27.5$ in the placebo group (difference of 8.9 (95\% CI 0.2-17.6); $p=0.052$ ). Mean duration of vasopressor therapy and average fluid balance were non-significant. See Supplement Table 4A for further details. 
A

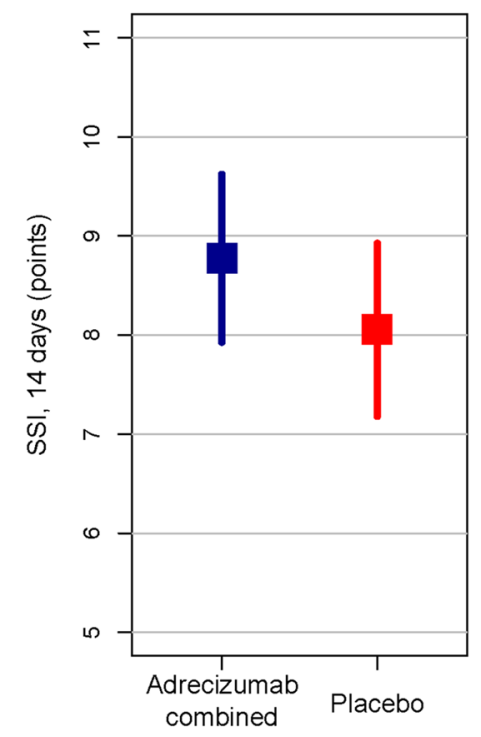

B

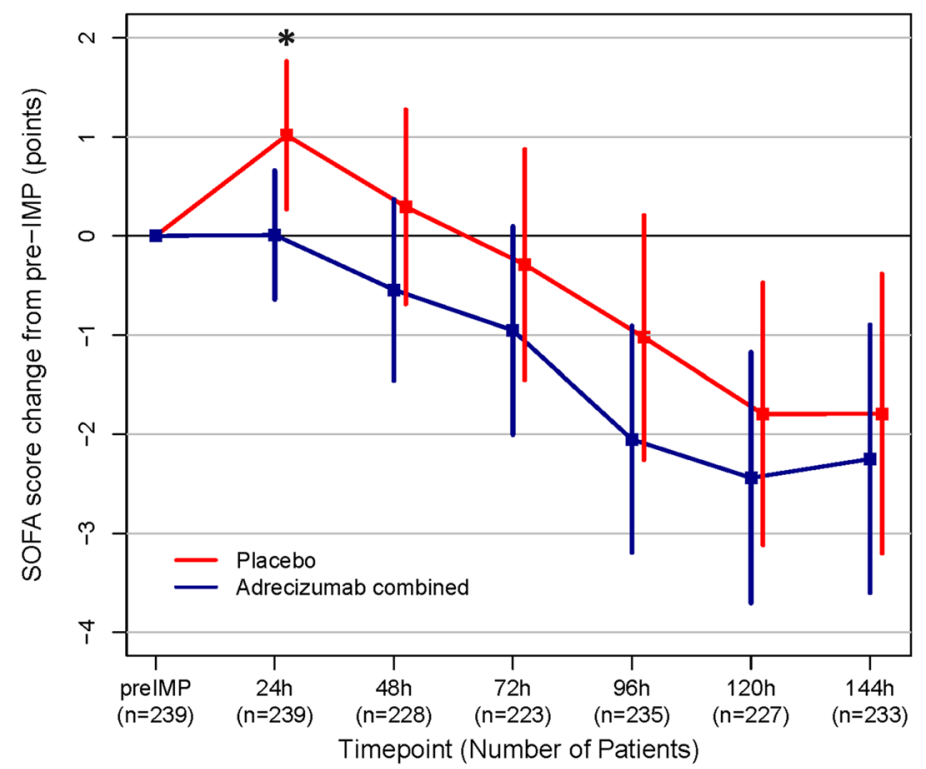

Fig. 3 Efficacy endpoints in the ITT population_SSI and SOFA score change. A Mean and 95\% Cl of Sepsis Support Index (SSI) with 14 days followup, Adrecizumab combined (blue) and placebo (red) for ITT ( $p=0.32$, Kruskal-Wallis test, and $p=0.45$, two-sample Kolmogorov-Smirnov test). B SOFA score change (points) starting prior Adrecizumab/placebo administration (pre-Investigational Medicinal Products (IMP)) (mean with 95\% Cl) for ITT ( $n=254$ with pre-IMP SOFA score available). For each time point the maximum number of patients with available SOFA score data was used. All time points show the same directionality. ${ }^{*} p<0.05$ for $24 \mathrm{~h}$ change. As the number of missing patients differed between time points, post hoc sensitivity analysis were performed and results shown in supplement Fig. 6

A

$\begin{array}{llll}\text { analysis } & \text { Dose } & \text { n } & \text { event } \\ \text { ITT } & \text { combined } & \mathbf{3 0 1} & \mathbf{7 7} \\ & 2 \mathrm{mg} / \mathrm{kg} & 224 & 60 \\ & 4 \mathrm{mg} / \mathrm{kg} & 229 & 59 \\ & & & \\ \text { bio-ADM adj. } & \text { combined } & \mathbf{2 9 8} & \mathbf{7 6} \\ & 2 \mathrm{mg} / \mathrm{kg} & 223 & 60 \\ & 4 \mathrm{mg} / \mathrm{kg} & 227 & 58 \\ & & & \\ \text { sOFA adj. } & \text { combined } & \mathbf{2 5 4} & \mathbf{6 7} \\ & 2 \mathrm{mg} / \mathrm{kg} & 193 & 55 \\ & 4 \mathrm{mg} / \mathrm{kg} & 193 & 50\end{array}$

0.899

0.899

0.781

0.763
0.841

0.696

0.724

0.863

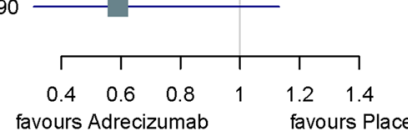

B

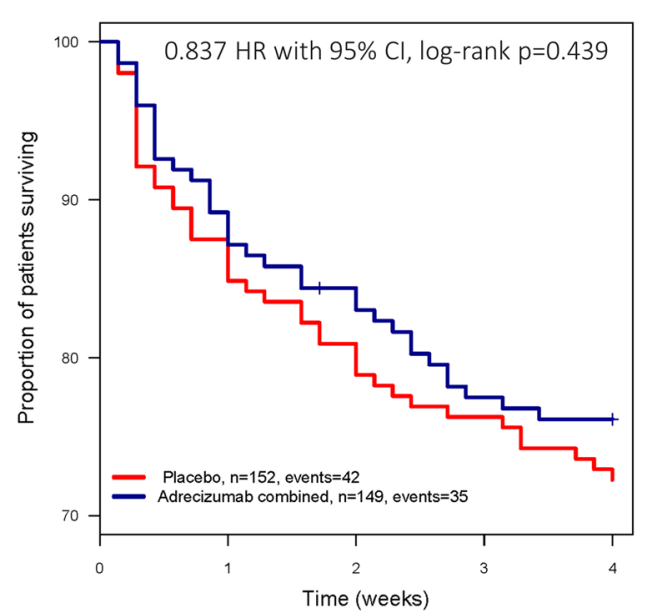

Fig. 4 Efficacy endpoints in the ITT population-hazard ratios (HR) and survival rates. A HR with 95\%-Cl for 28-day mortality $(n=301)$ Combined $=$ both doses of adrecizumab + the placebo group as control; $2 \mathrm{mg} / \mathrm{kg}=$ treatment arm with $2 \mathrm{mg} / \mathrm{kg}$ adrecizumab + the placebo group as control; $4 \mathrm{mg} / \mathrm{kg}$ = treatment arm with $4 \mathrm{mg} / \mathrm{kg}$ adrecizumab + the placebo group as control. Treatment effects were adjusted for initial severity: baseline bio-ADM (bio-ADM adj.); pre-IMP SOFA score (Score prior adrecizumab administration; SOFA adj.)—-to rule out baseline differences affecting the observed mortality reduction. B Kaplan-Meier plot for 28-day mortality: adrecizumab combined and placebo $(n=301)$

\section{Per-protocol analyses}

Seven patients had to be excluded for the PP analysis, details are listed in Supplement Table 2. Patient characteristics for the PP population can be found in Supplement Table 3. Safety results were similar to those in ITT (Supplement Table 1), and efficacy results tended to move in favor of adrecizumab (see 
Supplement Figs. 3-5 and Supplement Table 4B for details).

\section{Dose effect}

With respect to safety, no apparent dosing effect could be observed: In the adrecizumab $2 \mathrm{mg} / \mathrm{kg}$ group, $51 / 72$ (70.8\%) patients and in the adrecizumab $4 \mathrm{mg} / \mathrm{kg}$ group, 54/77 (70.1\%) patients experienced a TEAEs of grade 3 or higher. TEAE's related to the drug administration were reported in in $6(8.3 \%)$ patients in the adrecizumab $2 \mathrm{mg} / \mathrm{kg}$ group and $6(7.8 \%)$ patients in the adrecizumab $4 \mathrm{mg} / \mathrm{kg}$ group (Fig. 2).

\section{Pharmacokinetics}

In the pharmacokinetic study data set $(n=53$ for whom demographics were similar to the entire studied population, (see electronic supplementary material), the mean peak plasma concentrations $\left(C_{\max }\right)$ for free adrecizumab in patients treated with $2 \mathrm{mg} / \mathrm{kg}$ adrecizumab was $38.2 \mu \mathrm{g} / \mathrm{mL}$ (SD 10.4) and $86.9 \mu \mathrm{g} / \mathrm{mL}$ (SD $22.2)$ in $4 \mathrm{mg} / \mathrm{kg}$ group. Terminal half-life was similar in the two adrecizumab-treated groups: 206 (SD 44) hours versus 178 (SD 44) hours in adrecizumab $2 \mathrm{mg} /$ $\mathrm{kg}$ and $4 \mathrm{mg} / \mathrm{kg}$, respectively. As the bio-ADM concentration slowly decreased, the levels of circulating MR-proADM, the stable fragment of pro-ADM, were similar among the three groups (Fig. 5).

\section{Discussion}

The AdrenOSS-2 trial is the first randomized controlled trial in the ICU to be completed using a study-specific biomarker related to the proposed mechanism of action to select trial participants. Septic shock patients suffering from endothelial barrier dysfunction as manifested by elevated bio-ADM levels were randomized for the treatment with the anti-ADM antibody adrecizumab, modulating the ADM pathway, or placebo in a phase II clinical trial. Adrecizumab at both tested doses ( 2 or $4 \mathrm{mg} / \mathrm{kg}$ ) achieved the primary endpoint, as it was well tolerated and revealed a favorable safety profile as the prevalence of all recorded safety parameters were similar between adrecizumab and placebo groups.

Adrecizumab as a high-affinity, but non-neutralizing antibody is thought to enhance the endogenous plasma bio-ADM activity, exerting beneficial effects on the endothelium, while at the same time limiting the unfavorable effects of bio-ADM on the vascular smooth muscle cells [10]. Increase in circulating bio-ADM might result from (a) hypothesized partial redistribution of bio-ADM from the interstitium-where it may trigger vasodilation via the vascular smooth muscle cellstowards the lumen of the vessels, and (b) the reduction of proteolytic degradation of plasma bio-ADM [10]. In agreement with confinement in the circulation, in the present study, we observed a higher initial concentration $\left(C_{\max }\right)$ of adrecizumab in the $4 \mathrm{mg} / \mathrm{kg}$ group compared to $2 \mathrm{mg} / \mathrm{kg}$ and adrecizumab exhibited a terminal elimination half-life of 7-8 days. Based on the present result, further clinical steps will use $4 \mathrm{mg} / \mathrm{kg}$ of adrecizumab. In addition, we confirmed a dose-dependent adrecizumabinduced increase of plasma bio-ADM with no change in MR-proADM, demonstrating that the bio-ADM increase is not due to de novo synthesis, as observed previously in phase I clinical trials [19]. As other therapeutic antibodies and proteins also show a similar accelerated clearance in sepsis or severe inflammatory disease, a general mechanism of enhanced elimination is obvious and this could be, at least partially, an accelerated intracellular degradation. The concentration of free adrecizumab might be reduced by other mechanisms as well, including
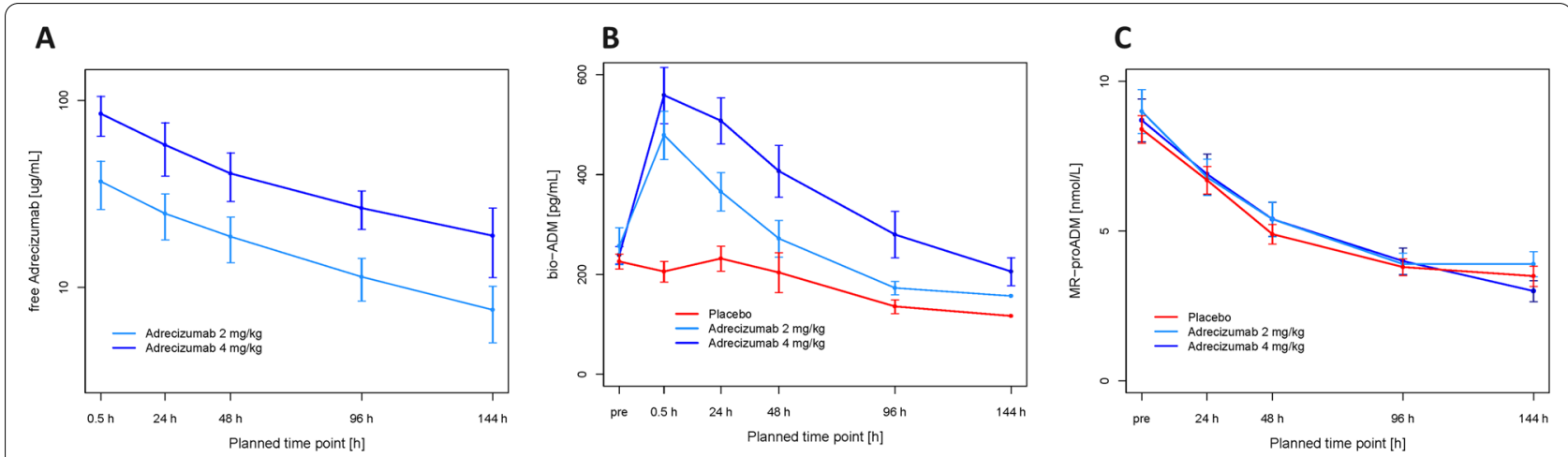

Fig. 5 Pharmacokinetics by time of blood draw and treatment arm, $\mathbf{A}$ geometric means ( \pm Standard Deviation (SD) of free adrecizumab in $n=53$ patients (eligible PK analysis set), B means of bio-ADM ( \pm standard error of the mean (SEM)) (all available samples), and C MR-proADM ( \pm standard error of the mean) (all available samples) 
a disease-related augmented production rate of its antigen ligand bio-ADM.

AdrenOSS-2 also explored signals of clinical efficacy of adrecizumab in this biomarker-guided trial. The SSI, a novel composite endpoint aiming at integrating both, mortality and organ dysfunction [17], showed no difference among studied groups while a rapid and consistent reduction of SOFA score was observed in adrecizumabtreated patients compared to placebo. In adrenOSS-2, SSI was similar in some aspects to changes in SOFA score, though differed in others, including the weight of mortality. In one hand, the advantage of the SSI was that it relied on fewer data points than the SOFA score, and was, therefore, easier to record. In the other hand, SOFA score typically has missing data in up to $10-20 \%$ of patients on each specific day despite efforts to get complete recordings. Though post hoc sensitivity analysis showed similar results, missings and methods to handle them confer important bias. Another difference between the 2 scores is that, change in SOFA took into account the severity of a patient on inclusion, therefore, measured relative change while SSI can only observe absolute change per treatment group. Finally, the SSI incorporates three organ classes, and mortality, in a dichotomous count, while the SOFA score incorporates six organ classes and each can change on a scale from 0 to 4 . Accordingly, based on AdrenOSS-2 trial and on recent recommendations to improve clinical trial design in critical care [16], the next phase IIb/III of the adrecizumab program, named ENCOURAGE, will assess adrecizumab $(4 \mathrm{mg} / \mathrm{kg})$ in septic shock patients, very early after initiation of vasopressor, combining predictive and prognostic enrichment strategies and targeting benefits on 28-day mortality and change in SOFA with special attention in preventing missing values.

The limitations of our trial include the lack of power to detect clinically important differences or to define dose response. Both doses turned out to be safe with a good tolerability profile, therefore both doses might be considered for future biomarker-guided trials. Further improvement may be achieved by enrichment of the patient group that may benefit most and an optimized treatment window for septic shock to further enhance the survival rate. Our study therapy was initiated within the first $12 \mathrm{~h}$ after detection of septic shock, a time-span found to be decisive for initiation of specific therapy. Secondary endpoints were not adjusted for multiple testing, therefore some results may have become significant by chance and results need replication/confirmation.

In conclusion, AdrenOSS-2 demonstrated that the novel trial design with a personalized approach applying a pathway-related biomarker was duable, even in the stringent ICU setting. Furthermore, this contemporary biomarker-guided phase 2 trial in septic shock demonstrated that there were no overt signals of harm and rather good tolerance with the use of adrecizumab. Benefits should yet be assessed in future biomarker-designed trials.

\section{Supplementary Information}

The online version contains supplementary material available at https://doi. org/10.1007/s00134-021-06537-5.

\section{Author details}

${ }^{1}$ Cliniques Universitaires Saint-Luc, (UCL Bruxelles), Avenue Hippocrate 10, 1200 Brussels, Belgium. ${ }^{2}$ Radboud University Medical Center, Geert Grooteplein Zuid 10, 6525 GA Nijmegen, The Netherlands. ${ }^{3}$ Klinik fur Operative Intensivmedizin und Intermediate Care, Universitätsklinikum Aachen, Pauwelsstraße 30, 52074 Aachen, Germany. ${ }^{4}$ Critical Care Department, Cliniques Universitaires St Luc, UC Louvain, Avenue Hipporcrate 10, 1200 Brussels,

Belgium. ${ }^{5}$ Faculté de Médecine, Service de Médecine Intensive-Réanimation, Université de Strasbourg (UNISTRA), Hôpitaux Universitaires de Strasbourg, Nouvel Hôpital Civil, Strasbourg, France. ${ }^{6}$ INSERM (French National Institute of Health and Medical Research), UMR 1260, Regenerative NanoMedicine (RNM), Fédération de Médecine Translationnelle (FMTS), University of Strasbourg, Strasbourg, France. ${ }^{7}$ Clinique St. Pierre, Avenue Reine Fabiola 9, 1340 Ottignies, Belgium. ${ }^{8}$ Intensive Care, $\mathrm{CH}$ Jolimont, Hospitalier de Jolimont, Rue Ferrer 159, 7100 Haine Saint Paul, Belgium. ${ }^{9}$ Klinik und Poliklinik fur Anaesthesiologie und Intensivtherapie, Universitätsmedizin Rostock, Schillingallee 35, 18057 Rostock, Germany. ${ }^{10}$ Réanimation Polyvalente and Inserm CIC1435 \& UMR1092, CHU de Limoges, France. ${ }^{11} \mathrm{CHU}$ de Nantes, Médecine Intensive Réanimation, 30 Bd. Jean Monnet, 44093 Nantes Cedex 1, France. ${ }^{12}$ Intensive Care Center, Medisch Spectrum Twente, Koningsplein 1,7512KZ Enschede, The Netherlands. ${ }^{13}$ Service d'Anesthésie-Réanimation, Hôpital Saint-Louis, 1 Avenue Claude Vellefaux, 75010 Paris, France. ${ }^{14} \mathrm{CH}$ Victor Dupouy, 69 Rue du Lieutenant-Colonel Prud'hon, 95107 Argenteuil Cedex, France. ${ }^{15}$ Canisius-Wilhelmina-Ziekenhuis (CWZ), Weg door Jonkerbosch 100, 6532 SZ Nijmegen, The Netherlands. ${ }^{16}$ CHD-Vendée, Médecine Intensive Réanimation, Boulevard Stéphane Moreau, 85000 La Roche-Sur-Yon, France. 17 Université de Paris, U942 Inserm, MASCOT, Hôpitaux Universitaires SaintLouis- Lariboisière, Fernand-Widal, 2, rue Ambroise-Paré, 75475 Paris Cedex 10, France. ${ }^{18}$ Fédération de Médecine Translationnelle de Strasbourg, Hôpitaux Universitaires de Strasbourg, Service d'Anesthésie-Réanimation \& Médecine Péri-Opératoire-Université de Strasbourg, EA3072 Strasbourg, France.

${ }^{19}$ Department of Anesthesiology and Intensive Care Medicine, Jena University Hospital, Am Klinikum 1, 07747 Jena, Germany. ${ }^{20}$ Center for Sepsis Control and Care (CSCC), Jena University Hospital, Jena, Germany. ${ }^{21}$ Département Anesthésie et Réanimation, CHU de Clermont-Ferrand, Pôle de Médecine Péri-opératoire, Clermont-Ferrand, France. ${ }^{22}$ Department of Intensive Care Medicine, University Medical Center Hamburg-Eppendorf, Hamburg, Germany. ${ }^{23}$ Faculté de Médecine, Service de Médecine Intensive-Réanimation, Université de Strasbourg (UNISTRA), Hôpitaux Universitaires de Strasbourg, Nouvel Hôpital Civil, Strasbourg, France. ${ }^{24}$ Adrenomed AG, Neuendorfstr. 15a, 16761 Hennigsdorf, Germany. ${ }^{25}$ Université de Paris, U942 Inserm, MASCOT, APHP, Fédération Hospitalo-Universitaire PROMICE, Hôpitaux Universitaires Saint-Louis-Lariboisière, Fernand-Widal, 2, Rue Ambroise-Paré, 75475 Paris Cedex 10, France. ${ }^{26}$ Département d'Anesthésie-Réanimation, Hôpital Lariboisière, 2 Rue A Paré, 75010 Paris, France.

\section{Acknowledgements}

The authors would like to thank, Deborah Bergmann, Karine Bourgeois, Ruxandra Lenz and Claudia Richter for their review, scientific input and substantial support to this paper. The AdrenOSS-2 study participants: Diego Castanares (Cliniques Universitaires Saint-Luc, (UCL Bruxelles), Avenue Hippocrate 10, 1200 Brussels, Belgium), Christine Collienne (Cliniques Universitaires Saint-Luc, (UCL Bruxelles), Avenue Hippocrate 10, 1200 Brussels, Belgium), Ludovic

Gèrard (Cliniques Universitaires Saint-Luc, (UCL Bruxelles), Avenue Hippocrate 10, 1200 Brussels, Belgium), Phillipe Hantson (Cliniques Universitaires SaintLuc, (UCL Bruxelles), Avenue Hippocrate 10, 1200 Brussels, Belgium), Virginie Montiel (Cliniques Universitaires Saint-Luc, (UCL Bruxelles), Avenue Hippocrate 10, 1200 Brussels, Belgium), Caroline Berghe (Cliniques Universitaires Saint-Luc, 
(UCL Bruxelles), Avenue Hippocrate 10, 1200 Brussels, Belgium), Marie-France Dujardin (Cliniques Universitaires Saint-Luc, (UCL Bruxelles), Avenue Hippocrate 10, 1200 Brussels, Belgium), Leslie Gielens (Cliniques Universitaires SaintLuc, (UCL Bruxelles), Avenue Hippocrate 10, 1200 Brussels, Belgium), Suzanne Renard (Cliniques Universitaires Saint-Luc, (UCL Bruxelles), Avenue Hippocrate 10, 1200 Brussels, Belgium), Philippe Jorens (Antwerp University, Hospital (UZA), Critical Care Medicine, Clinical Pharmacology/Toxicology, Wilrijkstraat 10, 2650 Edegem, Belgium), Pierre Asfar (CHU Angers Centre, Hospitalier Universitaire Département de Médecine Intensive 4, rue Larrey, 49933 Angers Cedex 9, France), Gaëtan Plantefève (CH Victor Dupouy, Service de Réanimation polyvalente et USC, 69 Rue du Lieutenant-Colonel Prud'hon, 95107 Argenteuil Cedex, France), Jacques Duranteau (Hôpital de Bicêtre, Service d'anesthésie-réanimation chirurgicale Bâtiment Paul Broca 78, rue du Général Leclerc, 94270 Le Kremlin-Bicêtre, France), Emmanuel Weiss (Hôpital Beaujon Anesthésie Réanimation Hôpitaux Universitaires Paris, Nord Val de Seine 100 bd du Général Leclerc, 92118 Clichy Cedex, France), Constance Vuillard (Hopital Louis Mourier Réanimation Médicale, 178 rue des Renoulliers, 92700 Colombes, France), Anne-Laure Fedou (CHU de Limoges Réanimation Polyvalente, 2 avenue Martin-Luther King, 87042 Limoges cedex, France), Marine Goudelin (CHU de Limoges Réanimation Polyvalente, 2 avenue Martin-Luther King, 87042 Limoges cedex, France), Bruno Evrard (CHU de Limoges Réanimation Polyvalente, 2 avenue Martin-Luther King, 87042 Limoges cedex, France), Thomas Daix (CHU de Limoges Réanimation Polyvalente, 2 avenue MartinLuther King, 87042 Limoges cedex, France), Arnaud Desachy (CHU de Limoges Réanimation Polyvalente, 2 avenue Martin-Luther King, 87042 Limoges cedex, France), Philippe Vignon (CHU de Limoges Réanimation Polyvalente, 2 avenue Martin-Luther King, 87042 Limoges cedex, France), Anne-Aurore Duchambon (CHU de Limoges Réanimation Polyvalente, 2 avenue Martin-Luther King, 87042 Limoges cedex, France), Ludmila Baudrillart (CHU de Limoges Réanimation Polyvalente, 2 avenue Martin-Luther King 87042 Limoges cedex, France), Paul Bourzeix (CHU de Limoges Réanimation Polyvalente, 2 avenue Martin-Luther King, 87042 Limoges cedex, France), Alexandra Gay (CHU de Limoges Réanimation Polyvalente, 2 avenue Martin-Luther King, 87042 Limoges cedex, France), Céline Prevost (CHU de Limoges Réanimation Polyvalente, 2 avenue Martin-Luther King, 87042 Limoges cedex, France), Coralie Chalot (CHU de Limoges Réanimation Polyvalente, 2 avenue Martin-Luther King, 87042 Limoges cedex, France), Isabelle Herafa (CHU de Limoges Réanimation Polyvalente, 2 avenue Martin-Luther King, 87042 Limoges cedex, France), Perrine Engels (CHU de Limoges Réanimation Polyvalente, 2 avenue MartinLuther King, 87042 Limoges cedex, France), Martin Maëlle (CHU de Nantes Médecine Intensive Réanimation, 30 Bd. Jean Monnet, 44093 Nantes cedex 1. France), Lila-Fariza Abeud (Hôpital Saint-Louis Service d'Anesthésie-Réanimation Plot A - 1er étage 1, avenue Claude Vellefaux, 75010 Paris, France), Laure Berton (Hôpital Lariboisière, 2 rue Ambroise Paré, 75010 Paris, France), Kamile Cerlinskaite (Hôpital Saint-Louis Service d'Anesthésie-Réanimation Plot A - 1er étage 1, avenue Claude Vellefaux, 75010 Paris, France), Nicolas Deye (Hôpital Lariboisière, 2 rue Ambroise Paré, 75010 Paris, France), Marie-Celine Fournier (Hôpital Lariboisière, 2 rue Ambroise Paré, 75010 Paris, France), Tassadit Hadjam (Hôpital Lariboisière, 2 rue Ambroise Paré, 75010 Paris, France), Alexa Hollinger (Hôpital Saint-Louis Service d'Anesthésie-Réanimation Plot A - 1er étage 1, avenue Claude Vellefaux, 75010 Paris, France), Tuija Javanainen (Hôpital Lariboisière, 2 rue Ambroise Paré, 75010 Paris, France), Clement Jourdaine (Hôpital Lariboisière, 2 rue Ambroise Paré, 75010 Paris, France), Matthieu Legrand (Hôpital Saint-Louis Service d'Anesthésie-Réanimation Plot A - 1er étage 1, avenue Claude Vellefaux, 75010 Paris, France), Badr Louadah (Hôpital Lariboisière, 2 rue Ambroise Paré, 75010 Paris, France), Arthur Neuschwander (Hôpital Européen Georges Pompidou Service d'Anesthésie-Réanimation Chirurgicale Université Paris Descartes 20 rue Leblanc, 75015 Paris, France), Raphaël Clere-Jehl (Nouvel Hôpital Civil Service de Réanimation Médicale 1, place de l'hôpital, 67091 Strasbourg, France), Julien Demiselle (Nouvel Hôpital Civil Service de Réanimation Médicale 1, place de l'hôpital, 67091 Strasbourg, France), Hamid Merdji (Hôpital de Hautepierre Hôpitaux Universitaires de Strasbourg Unité de Réanimation Chirurgicale Service d'Anesthésie-Réanimation Chirurgicale 1, Avenue Molière, 67200 Strasbourg, France), Alexandra Monnier (Nouvel Hôpital Civil Service de Réanimation Médicale 1, place de I'hôpital, 67091 Strasbourg, France), Emmanuelle Mercier (CHRU Bretonneau Medecine Intensive Réanimation 2, Boulevard Tonnelé, 37044 Tours cedex 9, France), Stefan Kluge (Universitätsklinikum Hamburg-Eppendorf Klinik für Intensivmedizin Ost 10, 2.OG, Postfach 381 Martinistraße 52, 20246 Hamburg, Germany), Alexander Zarbock (Universitätsklinikum Münster Klinik für Anästhesiologie, operative Intensivmedizin und Schmerztherapie
Albert-Schweitzer-Campus 1, Gebäude A1 48149 Münster, Germany), Arthur R. H. van Zanten (Gelderse Vallei Hospital Department of Intensive Care Willy Brandtlaan 106716 RP Ede, Netherlands), Wytze Vermeijden (Medisch Spectrum Twente Department of Intensive Care Koningsplein 17512 KZ Enschede, Netherlands), Tom Dormans (Zuyderland Medical Center Department of Intensive Care H. Dunantstraat 56401 CX Heerlen, Netherlands).

\section{Funding}

The AdrenOSS-2 study was funded by Adrenomed AG.

\section{Declarations}

\section{Conflicts of interest}

P-F $L$ received fees as a coordinator of the CCC. PP reports travel and consultancy reimbursement from Adrenomed, SphingoTec, 4TEEN4, AM-Pharma, Baxter, EBI. XW was part of the Clinical Coordinating Center assessing patient's eligibility. No other conflict of interest. FM has no conflict of interest. TD received fees as a coordinator of the CCC. BF reports personal fees outside the submitted work from Inotrem, Biomérieux, AM-Pharma, Takeda, Enlivex, Aridis, GSK, Asahi-Kasai. JBL reported receiving consultation fees from Asahi Kasei America Corporation outside the submitted work. AB Consultancy fees from GSK and Danone Nutricia. DC has no conflict of interest. OH has no conflict of interest. JCL has no conflict of interest to declare. BC served as a member of an advisory board for Roche Diagnostics. JP has no conflict of interest with the present work. MB holds shares of SmartDyeLivery GmbH, Jena. He has received funding for scientific advisory boards, travel and speaker honoraria by T2 Biosystems, Inc., La Jolla Pharmaceutical Company, SNIPR BIOME Denmark, CytoSorbents GmbH, Thermo Fisher Scientific (B.R.A.H.M.S GmbH), Roche Diagnostics International Ltd., Transgene S.A. and SphingoTec GmbH. TG has non conflict of interest with the present work. MK reports grant support from Adrenomed and Vifor, and honoraria from Adrenomed, SphingoTec, Vifor, Amgen, 4TEEN4, Astra-Zeneca, and Sanofi. AM reports personal fees from Orion, Sanofi, Adrenomed, Epygon and Fire 1 and grants and personal fees from 4TEEN4, Abbott, Roche and SphingoTec.

\section{Publisher's Note}

Springer Nature remains neutral with regard to jurisdictional claims in published maps and institutional affiliations.

Received: 12 May 2021 Accepted: 9 September 2021 Published online: 4 October 2021

\section{References}

1. Singer M, Deutschman CS, Seymour CW, Shankar-Hari M, Annane D, Bauer M, Bellomo R, Bernard GR, Chiche JD, Coopersmith CM et al (2016) The third international consensus definitions for sepsis and septic shock (Sepsis-3). JAMA 315(8):801-810

2. Lee WL, Slutsky AS (2010) Sepsis and endothelial permeability. N Engl J Med 363(7):689-691

3. Karpinich NO, Hoopes SL, Kechele DO, Lenhart PM, Caron KM (2011) Adrenomedullin function in vascular endothelial cells: insights from genetic mouse models. Curr Hypertens Rev 7(4):228-239

4. Hofbauer KH, Schoof E, Kurtz A, Sandner P (2002) Inflammatory cytokines stimulate adrenomedullin expression through nitric oxide-dependent and -independent pathways. Hypertension 39(1):161-167

5. Hofbauer KH, Jensen BL, Kurtz A, Sandner P (2000) Tissue hypoxygenation activates the adrenomedullin system in vivo. Am J Physiol Regul Integr Comp Physiol 278(2):R513-519

6. Shoji H, Minamino N, Kangawa K, Matsuo H (1995) Endotoxin markedly elevates plasma concentration and gene transcription of adrenomedullin in rat. Biochem Biophys Res Commun 215(2):531-537

7. Mebazaa A, Geven C, Hollinger A, Wittebole X, Chousterman BG, Blet A, Gayat E, Hartmann O, Scigalla P, Struck J et al (2018) Circulating adrenomedullin estimates survival and reversibility of organ failure in sepsis: the prospective observational multinational adrenomedullin and outcome in sepsis and septic shock-1 (AdrenOSS-1) study. Crit Care 22(1):354

8. Caironi P, Latini R, Struck J, Hartmann O, Bergmann A, Maggio G, Cavana M, Tognoni G, Pesenti A, Gattinoni L et al (2017) Circulating biologically 
active adrenomedullin (bio-ADM) predicts hemodynamic support requirement and mortality during sepsis. Chest 152(2):312-320

9. Kim H, Hur M, Struck J, Bergmann A, Di Somma S (2019) Circulating biologically active adrenomedullin predicts organ failure and mortality in sepsis. Ann Lab Med 39(5):454-463

10. Geven C, Bergmann A, Kox M, Pickkers P (2018) Vascular Effects of adrenomedullin and the anti-adrenomedullin antibody adrecizumab in sepsis. Shock 50(2):132-140

11. Geven C, Peters E, Schroedter M, Struck J, Bergmann A, McCook O, Radermacher P, Kox M, Pickkers P (2018) Effects of the humanized antiadrenomedullin antibody adrecizumab (HAM8101) on vascular barrier function and survival in rodent models of systemic inflammation and sepsis. Shock 50(6):648-654

12. Blet A, Deniau B, Geven C, Sadoune M, Caillard A, Kounde PR, Polidano E, Pickkers P, Samuel JL, Mebazaa A (2019) Adrecizumab, a non-neutralizing anti-adrenomedullin antibody, improves haemodynamics and attenuates myocardial oxidative stress in septic rats. Intensive Care Med Exp 7(1):25

13. Wagner K, Wachter U, Vogt JA, Scheuerle A, McCook O, Weber S, Groger M, Stahl B, Georgieff M, Moller P et al (2013) Adrenomedullin binding improves catecholamine responsiveness and kidney function in resuscitated murine septic shock. Intensive Care Med Exp 1(1):21

14. Thiele C, Simon TP, Szymanski J, Daniel C, Golias C, Hartmann O, Struck J, Martin L, Marx G, Schuerholz T (2020) Effects of the non-neutralizing humanized monoclonal anti-adrenomedullin antibody adrecizumab on hemodynamic and renal injury in a porcine two-hit model. Shock 54(6):810-818

15. Angus DC, van der Poll T (2013) Severe sepsis and septic shock. N Engl J Med 369(21):2063

16. Harhay MO, Casey JD, Clement M, Collins SP, Gayat É, Gong MN, Jaber S, Laterre PF, Marshall JC, Matthay MA et al (2020) Contemporary strategies to improve clinical trial design for critical care research: insights from the first critical care clinical Trialists workshop. Intensive Care Med 46(5):930-942

17. Geven C, Blet A, Kox M, Hartmann O, Scigalla P, Zimmermann J, Marx G, Laterre PF, Mebazaa A, Pickkers P (2019) A double-blind, placebocontrolled, randomised, multicentre, proof-of-concept and dose-finding phase II clinical trial to investigate the safety, tolerability and efficacy of adrecizumab in patients with septic shock and elevated adrenomedullin concentration (AdrenOSS-2). BMJ Open 0:e024475

18. de Grooth HJ, Geenen IL, Girbes AR, Vincent JL, Parienti JJ, Oudemansvan Straaten HM (2017) SOFA and mortality endpoints in randomized controlled trials: a systematic review and meta-regression analysis. Crit Care 21(1):38

19. Geven C, van Lier D, Blet A, Peelen R, Ten Elzen B, Mebazaa A, Kox M, Pickkers P (2018) Safety, tolerability and pharmacokinetics/pharmacodynamics of the adrenomedullin antibody adrecizumab in a first-inhuman study and during experimental human endotoxaemia in healthy subjects. Br J Clin Pharmacol 84(9):2129-2141 